\title{
B-meson Anomalies and New Physics for Flavor Violation
}

\author{
Soo-Min Choi, Yoo-Jin Kang, Hyun Min Lee, Tae Gyu Ro* \\ Department of Physics, Chung-Ang University, Seoul 06974, Korea \\ E-mail: soominchoigo@gmail.com, yoojinkang91@gmail.com, \\ hminleedcau.ac.kr, shxorb234@gmail.com
}

The LHCb experiment has recently provided several new measurements to test the lepton flavor universality in the Standard Model (SM) and confirmed some of the prevailing anomalies from the B-meson decays in BaBar and/or Belle experiments. We consider the setup where scalar leptoquarks have flavor-dependent couplings to the SM. In this work, we discuss the flavor structure for quarks and leptons and various constraints on the model and propose a natural candidate for dark matter.

The 39th International Conference on High Energy Physics (ICHEP2018)

4-11 July, 2018

Seoul, Korea

${ }^{*}$ Speaker. 
In the Standard Model, lepton flavor universality for weak interactions is confirmed by $\tau$ decay and $Z^{0}$ decay, etc. But, recent measurements on the semi-leptonic decays of $B$-mesons at BaBar, Belle and LHCb experiments raise intriguing anomalies, $R_{K^{(*)}}$ and $R_{D^{(*)}}$. The reported values of $R_{K^{(*)}}=\mathscr{B}\left(B \rightarrow K^{*} \mu^{+} \mu^{-}\right) / \mathscr{B}\left(B \rightarrow K^{*} e^{+} e^{-}\right)$are deviated from the SM prediction by $2.1-2.5 \sigma$. The results for $R_{D^{(*)}}=\mathscr{B}\left(B \rightarrow D^{*} \tau^{-} v_{\tau}\right) / \mathscr{B}\left(B \rightarrow D^{*} l^{-} v_{l}\right)$ with $l=e, \mu$ also show derivations from the SM prediction by $4 \sigma$ [2].

We consider the Lagrangian for an $S U(2)_{L}$ singlet scalar leptoquark $S_{1}$ with $Y=+\frac{1}{3}$, and an $S U(2)_{L}$ triplet scalar leptoquark $S_{3} \equiv \Phi_{a b}=\left(\begin{array}{cc}\sqrt{2} \phi_{3} & -\phi_{2} \\ -\phi_{2} & -\sqrt{2} \phi_{1}\end{array}\right)$ with $Y=+\frac{1}{3}$,

$$
\mathscr{L}_{L Q}=-\lambda_{i j} \overline{\left(Q^{C}\right)_{R i}^{a}}\left(i \sigma^{2}\right)_{a b} S_{1} L_{L j}^{b}-\kappa_{i j} \overline{\left(Q^{C}\right)_{R i}^{a}} \Phi_{a b} L_{L j}^{b}+\text { h.c. }
$$

By integrating out the leptoquarks $S_{1}$ and $S_{3}$, we obtain the effective Hamiltonian relevant for $b \rightarrow c \tau \bar{v}_{\tau}$ and $b \rightarrow s \mu^{+} \mu^{-}$as

$$
\mathscr{H}_{b \rightarrow c \tau \bar{v}_{\tau}, b \rightarrow s \mu^{+} \mu^{-}}^{S_{1}, S_{3}}=-\frac{\lambda_{33}^{*} \lambda_{23}}{2 m_{S_{1}}^{2}}\left(\bar{b}_{L} \gamma^{\mu} c_{L}\right)\left(\bar{v}_{\tau L} \gamma_{\mu} \tau_{L}\right)-\frac{\kappa_{32}^{*} \kappa_{22}}{m_{\phi_{1}}^{2}}\left(\bar{b}_{L} \gamma^{\mu} s_{L}\right)\left(\bar{\mu}_{L} \gamma_{\mu} \mu_{L}\right)+\text { h.c. }
$$

which explains the B-anomalies in the parameter space shown in figure. 1 (first and second plots).

We introduce a singlet real scalar dark matter $S$ with leptoquark $S_{L Q}$ and Higgs couplings,

$$
\begin{aligned}
\mathscr{L}_{S}= & \left|D_{\mu} S_{L Q}\right|^{2}-m_{L Q}^{2}\left|S_{L Q}\right|^{2}+\frac{1}{2}\left(\partial_{\mu} S\right)^{2}-\frac{1}{2} m_{S}^{2} S^{2} \\
& -\frac{1}{4} \lambda_{1} S^{4}-\lambda_{2}\left|S_{L Q}\right|^{4}-\frac{1}{2} \lambda_{3} S^{2}\left|S_{L Q}\right|^{2}-\frac{1}{2} \lambda_{4} S^{2}|H|^{2}-\lambda_{5}|H|^{2}\left|S_{L Q}\right|^{2} .
\end{aligned}
$$

In this model, we determine the dark matter relic density by the direct and cascade annihilations with leptoquarks. We show various constraints in the parameter space, $\lambda_{4}$ vs $m_{S}$, in figure 1 (third and fourth plots) [3]: direct detection bound (XENON1T) and indirect detection bound (Fermi-LAT, HESS and AMS-02). Additionally, Higgs data can constrain by using diphoton signal strength and Higgs invisible decay.
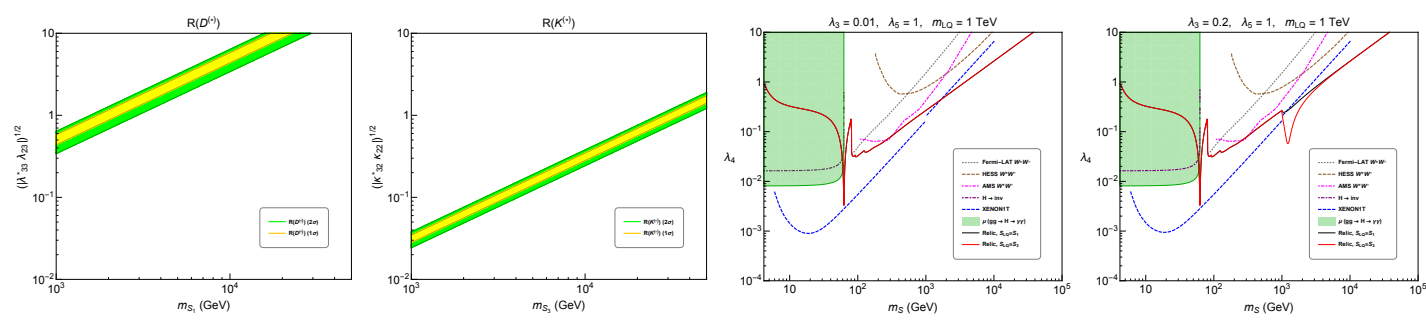

Figure 1: $R_{D^{(*)}}$ and $R_{K^{(*)}}$ anomalies (first and second), Parameter space, $\lambda_{4}$ vs $m_{S}$ (third and fourth)

\section{References}

[1] R. Aaij et al. [LHCb Collaboration], JHEP 1708 (2017) 055, [arXiv:1705.05802 [hep-ex]]

[2] Y. Amhis et al. [HFLAV Collaboration], Eur. Phys. J. C 77 (2017) no.12, 895, [arXiv:1612.07233 [hep-ex]]

[3] S. M. Choi, Y. J. Kang, H. M. Lee, T. G. Ro, JHEP 1810 (2018) 104， [arXiv:1807.06547 [hep-ph]] 